\title{
Amazing! Two Graduate Students Wrote About Stupid Internet Stuff and Got Away with It! (Page 10 Will Blow Your Mind!)
}

\author{
Zachary Sheldon, M.A. \\ Texas A\&M University, USA \\ (D) 0000-0003-3812-2882 \\ zsheldon@tamu.edu
}

Max Romanowski, M.A.

Baylor University, USA

(D) 0000-0002-5159-9370

Max_Romanowski@Baylor.edu

ARTICLE INFO

Received: 28 August 2018

Accepted: 25 October 2018

Published: 12 November 2018

DOI: https://doi.org/10.12973/ojcmt/3953

ABSTRACT

This article examines new forms of narrative in the internet age through examining the website ClickHole. Through parodying and satirizing sites like BuzzFeed, ClickHole (an offshoot of the famous parody entity The Onion) thrives on the creation and dissemination of absurd headlines and accompanying stories. These not only commenting widely on cultural obsessions with things like pop culture figures and social causes, but also point to an innovative take on the place and form of narrative in the age of the internet.

Keywords: ClickHole, BuzzFeed, internet culture, satire, new media, storytelling

\section{INTRODUCTION}

Founded in 2014 as an offshoot of popular satire The Onion, ClickHole is a "website with content unabashedly meant to pander, mislead and ultimately go viral" (Greenberg, 2014). Begun as The Onion was ceasing its print publication, ClickHole is a phenomenon entirely of the internet, created to comment on "the viral, click bait culture that's made BuzzFeed and Upworthy thrive" (Greenberg, 2014). But the site has not just stopped there. Though initially conceived as an explicit parody and satire of the headlines and stories found on sites like BuzzFeed, ClickHole has evolved to stand on its own as a unique, pure artifact of internet culture.

The early history of the internet was inextricably bound to the media that had come before, including webpages that were static and text-heavy, mimicking the form of newspapers and books. Over time users and developers have figured out the unique strengths afforded by the web's capabilities, and as the literal form and content of the internet have developed, so too has the actual culture of the internet such that the internet can be considered a distinctive culture. One prominent player in the development of this culture was the website BuzzFeed, which began as an aggregating news organization that quickly developed a new way of delivering content for ease of discovery and shareability. BuzzFeed took clear advantage of the internet's ability to make story 
content more public than it has ever been before, enabling even amateur writers to publish and reach wider audiences in a way only digital modes of storytelling could offer (Green \& Walters, 2013). Specifically highlighting discovery and shareability in the production of news meant that the complexity of actual story content was reduced, leading to sensationalized headlines designed to pique curiosity and evoke quick emotion to drive traffic to stories, no matter how insignificant the story actually was. This phenomenon gave rise to the term "clickbait," which implies that a story has no real value but is designed purely to attract clicks and thus increase advertising revenue ("clickbait I Definition of clickbait in English by Oxford Dictionaries,” n.d.). BuzzFeed's early success with such tactics inspired a host of imitators, leading to the spread of the term and to many decrying the state of information and journalism on the internet (Tandoc \& Jenkins, 2017).

ClickHole's adoption of a name so similar to this concept is no accident. The website's mission statement is explicit about their intent: "All content deserves to go viral" ("What Is ClickHole?," 2014). By choosing to blatantly critique the modern internet clickbait culture, ClickHole inadvertently raises the question of the role of narrative in the contemporary internet age--not only at the level of individual news stories, but also in regard to the structural narrative of websites and web culture. As a new medium, the internet calls for new ways to tell its stories.

This paper looks to examine how narrative is treated on the internet today, specifically focusing on BuzzFeed and ClickHole to articulate both the impact that BuzzFeed has had on our culture, and the ability for ClickHole as a website to critique that impact. Additionally, we argue that ClickHole has transcended its initial purpose as satire and has actually developed beyond those limitations to become the exemplar of the next phase of narrative on the internet. We begin by examining the history of BuzzFeed and its relationship to the internet and traditional media, which gave rise to the internet culture that enabled ClickHole to emerge. Following this, we analyze ClickHole to understand how it has developed a form of storytelling unique to the internet.

\section{BuzzFeed AND ClickHole}

BuzzFeed has something of a reputation on the internet. While the site has worked to rebrand itself as a legitimate journalistic enterprise through the hiring of former Politico reporter Ben Smith as editor-in-chief in 2011, BuzzFeed is still weighed down by its early reputation as a purveyor of inanity on the internet. Some see the site as directly responsible for the rise of "clickbait" and the overall dumbing down of audience expectations and comprehension on the internet (Fisher, n.d.; Hamblin, 2014; Jolles, 2014), a reputation that brings with it negative associations for those who share the site's content across their own social media. This is not to say that BuzzFeed does not deserve some credit for having reached the place they have. Established in 2006 by Jonah Peretti, one of the co-founders of Huffington Post, BuzzFeed was quickly recognized as an innovator in curating content seemingly tailor-made for sharing and discussing on the internet, including lists, short articles, and quizzes (Lafrance, 2012). ${ }^{1}$ And while content shared on the site was relatively unremarkable, its extensive reach across the internet led venture capital firm Andreessen Horowitz to value the company at US $\$ 850$ million in 2014 (Isaac, 2014).

\footnotetext{
${ }^{1}$ Noteworthy note is the irony of Peretti leaving Huffington Post to found a site that then impacted the evolution of Huffington Post, which recently rebranded itself as "HuffPost" and loosened content standards to a more BuzzFeed-like level.
} 
One of BuzzFeed's earliest innovations was the development of what became popularly known as the "listicle," which is exactly what it sounds like--"an article in the form of a list" (Okrent, 2014). With significantly varied subject matter--from "40 Of The Most Powerful Photographs Ever Taken" (Shepherd, 2012) to "15 Poop Horror Stories That Will Make You Feel Better About Yourself," (Yandoli, 2014), for instance--listicles established a quick, image and headline-heavy form that let readers quickly scroll through, get the point (or whatever they want to get out of the list), and move on. An additional factor that shows BuzzFeed's understanding of the internet is that many of these lists are comprised not of still images, but of looping GIFs that are oftentimes only tangentially related to the article's content. These articles proved to be remarkably popular and the form was quickly assimilated by reputable news organizations. ${ }^{2}$

The appeal of such a form is understandable in the age of the internet and the smartphone. Media scholars have argued that technology (and the internet specifically) has led to a way of thinking that encourages the quick acquisition of information over perhaps more meaningful, nuanced knowledge (Carr, 2011). Additionally, the physical form of smartphone screens and touch-based interfaces have privileged designs that enable easy scrolling to navigate through sites and information--hence the similarity in design between essentially all social network sites/apps, and the introduction of "Reader Modes" in mobile browsers that collapse content into an easy-to-scroll format. With the listicle, BuzzFeed recognized a gap between internet content and the form that it was taking, quickly establishing a new and oft-derided standard for how news and other stories ought to look, feel, and read for internet-age audiences.

Such a format is not without its consequences. Most notably, the meaningfulness of a story's content is often considered of lesser importance than the form that it takes. When headlines are designed both to capture attention and convey effectively all you need to know about a story, the informational content and its nuance is simply lost. News stories become informational tidbits rather than cohesive, full-fledged narratives. In terms of BuzzFeed's journalistic endeavors, this is a major departure from tradition. For decades journalists have followed the standard of the "Inverted Pyramid Structure," which places a story's most important and fundamental aspects near the top, with less essential information following in subsequent paragraphs ("The Inverted Pyramid Structure," n.d.). BuzzFeed's adoption of easy, attention-grabbing headlines and their use of bullet points as story content eschews detail in favor of ease of readability and comprehension.

Some of this has changed in recent years with BuzzFeed's hiring of Ben Smith as their editor-in-chief. Smith represents BuzzFeed's desire to be taken seriously as a news outlet, and some of the site has changed to reflect this intent, with "fluff and substance side by side" (Tandoc \& Jenkins, 2017, p. 485). This is prominently reflected in the layout of BuzzFeed's homepage, which "does not necessarily reflect editorial judgment in terms of story placement,"(Tandoc \& Jenkins, 2017, p. 489) but rather features a mixture of serious reporting and mindless lists often right next to each other, such that distinguishing legitimate news from entertainment is difficult. Many of these design and content decisions are indicative of BuzzFeed's fundamental content philosophy: according to Ze Frank, one of the managers in charge of BuzzFeed's video production wing, the only statistic that BuzzFeed cares about is the number of shares its content garners (Allocca, 2018, p. 42). He identifies shares as more difficult to accrue than simple content views, because an emotional response is required to spur audiences to share the content they are

\footnotetext{
${ }^{2}$ For just one meta example see (Edidin, 2014)
} 
viewing. Interestingly, Frank argues that one of the most unnecessary components in forming an emotional connection in a story is, ironically, story itself. Kevin Allocca's book on YouTube culture features an extended look at what this means and how it is made manifest in BuzzFeed's videos in particular and all their stories in general:

Instead of stories, they created a series of what Frank calls "moments"..."If we did a story, we would have one shot at hitting that line that gets [a viewer] to be like, 'Oh my god. That's totally me,' but if we did it in just a series of moments, we had all those shots and maybe three of them wouldn't connect but then that fourth one would be the zinger." (Allocca, 2018, p. 43)

As can be seen, BuzzFeed is replacing a traditional narrative that favors meaningful informational content with one that aims at virality and spreadability.

This is particularly interesting when considering the narrative of BuzzFeed's site. Here we would like to differentiate between the narrative of BuzzFeed as a company and the narrative promulgated by BuzzFeed's site. BuzzFeed's historical narrative begins with its founding and includes various events--like Smith's hiring--that bring it up the present day. The narrative of the site itself is its general reputation on the internet. This reputation is not dependent on the site's description of itself, but what emerges from the look, feel, and impressions of the site's content. Each story or piece on a particular website contributes to the site's overall vibe. Thus, BuzzFeed becomes defined by its listicles, absurd headlines, and its reputation for superficial reporting.

Understanding the narrative of an organization and their web presence in this way is not limited to sites like BuzzFeed. Serious newspapers like the Wall Street Journal or the New York Times may be considered in similar terms. Editorial boards and editors define a paper's style of reporting or their editorial or content standards, but the way this is meted out in individual stories varies significantly based on reporters' bias as well as the stories being told. The overall impression one builds of the New York Times and their web presence is defined by the reporting one finds on the site. This is what differentiates it from its competitors and establishes its status. The "macronarrative" of such sites emerges from the litany of micronarratives that make up the site's actual content. And while this model is not going to disappear anytime soon (Auwal, 2016), especially in relation to traditional media like newspapers, we argue that ClickHole actually introduces a new narrative structure for consideration.

As a direct satire of internet phenomena, ClickHole uproots and upends this narrative paradigm. Instead of the site's larger "macronarrative" emerging from many micronarratives, ClickHole is up front in its intentions: it has a grander vision driving its custom-developed content, so each individual story falls into a general narrative that the site is promoting. With a lack of explicit ties to telling stories rooted in the real world and current events, ClickHole is able to choose, control, and maintain its larger narrative through the pieces of content it produces and shares, much like a film director chooses the elements that comprise the individual shots that support the larger narrative and themes of a film. Unlike sites such as BuzzFeed where content is produced by a large number of contributors with a minimum of editorial input, ${ }^{3}$ everything at ClickHole is produced via a writer's room, much like most network sitcoms (Kois, 2015). This provides the site with a unified vision and voice, even amidst the peculiarity and diversity of its posts. Such policies position the site as pushing back against the tide of vapid content that emerges

${ }^{3}$ See (Allocca, 2018, pp. 40-42; Spangler, 2017) 


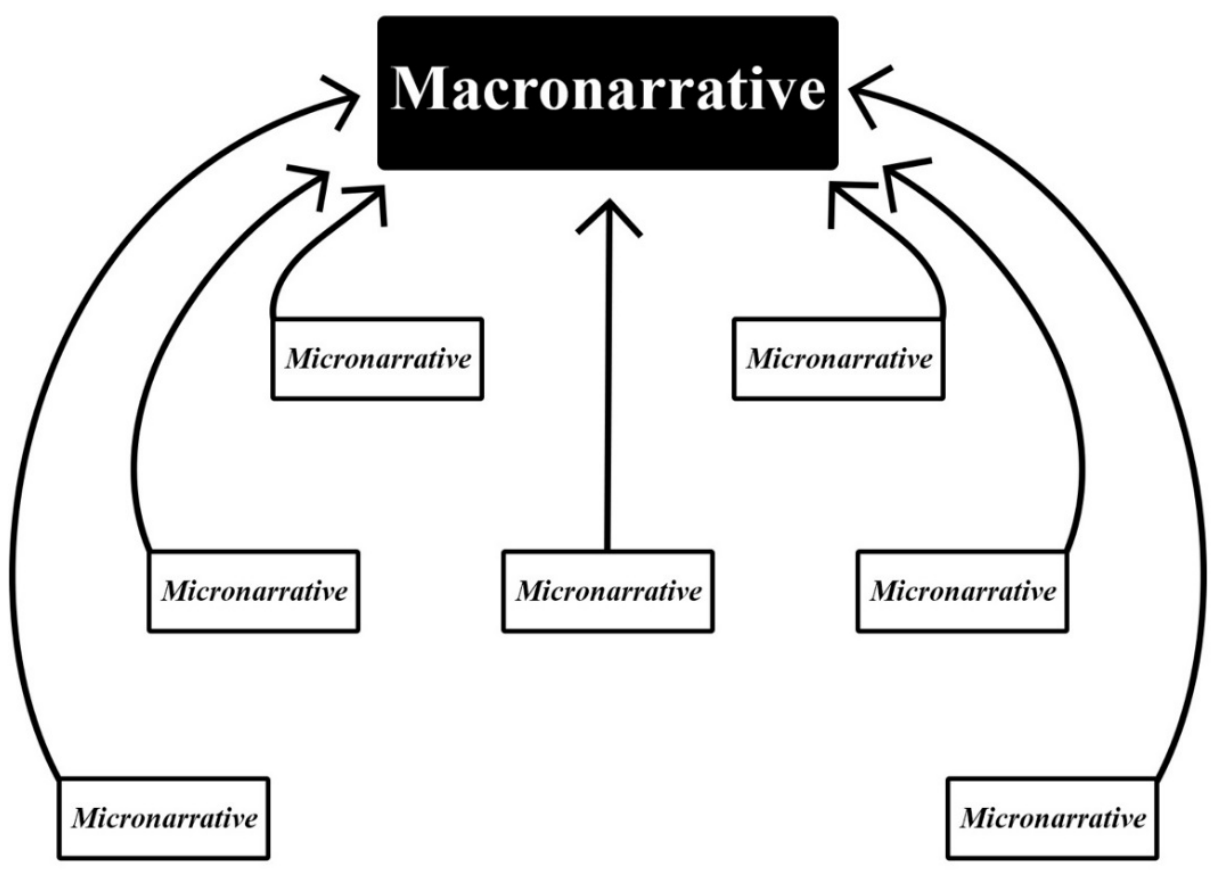

Figure 1. Traditional narrative structure

from other producers. This is, in part, what ClickHole's satirical wit is aimed towards. While their content may seem absurd and even nonsensical, there $i s$ actually something to it, as each piece--every video, article, or quiz--tells a part of the story that the site is communicating. This story, it may be argued, is actually the story of the internet itself.

ClickHole's site is divided into several sections of content: Lifestyle, News, Voices, Quizzes, Video, and ClickVentures. ${ }^{4}$ Below, we examine each of these categories to demonstrate how their unique content has emerged specifically from the internet's influence on the type of content generally found in stories associated with categories of these types.

\section{ClickHole's SIX CATEGORIES}

\section{Lifestyle}

One of the advantages of the internet as a whole is that on it anyone can have a voice. Of course, one consequence of everyone having the ability to publish their thoughts at will is that it lowers the bar for what may be considered noteworthy. One example of this is the rise of "lifestyle blogs," which typically features a single person whose posts center around their life and how they live it, such that anyone's life can be at the center of a lifestyle blog so long as you are willing to "own your lifestyle" (Lamar, 2015). 5 The lack of specificity in such a definition truly does mean that a lifestyle blog can be about anything. As a result, "lifestyle" content typically falls under an unusual umbrella of stories about people just doing things, some of which are interesting, some of which are remarkably trivial (Torres, 2018).

ClickHole takes this idea of triviality to its extreme. Lifestyle stories on the site often focus on what can only be considered to be the most mundane subjects--though each is

\footnotetext{
${ }^{4}$ There are actually two additional categories to be found on the site: PatriotHole and ResistanceHole, but these differ in branding by being notably more political and so remain unexamined here.

${ }^{5}$ Emphasis hers - both bolding and italicization.
} 
eminently relatable in some oddly specific way. Examples here include: "Whoa: Morgan Brought Coffee To First Period," and "This Bitch: Of Course Margot's Only Skin Routine Is Splashing Her Face With Water At Night" (ClickHole, "Whoa" 2018; ClickHole, "This Bitch" 2018). Additionally, the Lifestyle category leans into the fascination that many sites like BuzzFeed have with celebrity's, their lives, and their charitable works. This leads to headlines like "6 Times Tobey Maguire Had A Public Meltdown Because Nobody Would Call Him 'Tugboat Maguire," and "Hard To Watch: Chris Pine Accompanied This Terminally Ill Girl To Prom But Was Upstaged When Chris Pratt Accompanied An Even More Terminally Ill Girl To The Same Prom" (ClickHole, "6 Times Tobey Maguire Had A Public Meltdown Because Nobody Would Call Him 'Tugboat Maguire”" 2015; ClickHole, "Hard To Watch" 2017).

These examples show that ClickHole is rather overt in their lampooning of what people consider to be stories on the internet today. At the same time, as crazy at it is that there now exists a "story" about Tobey Maguire having six separate meltdowns about not being referred to as "Tugboat Maguire," this highlights that someone was also hired and paid to write a story about Amy Schumer answering fan questions while playing with puppies. ${ }^{6}$ While it is easy to see how over-the-top ClickHole's satire is, side-by-side comparisons of headlines from "real" news and lifestyle sites emphasizes the power of this satire by showing that even when the content of this form is pushed to its extreme it is still possible to confuse a fake headline with what many readers would consider to be a real one worthy of both time and consideration.

News

Media theorists have noted that the advent of electronic communication technologies like the telegraph began what many characterize as a downward spiral in what could be considered meaningful news (Boorstin, 2012; Postman, 1993). Where before, "news" was reserved to mean information that had a direct impact on people's lives, the telegraph, telephone, and other electronic media enabled the transmission of even relatively unimportant or unnecessary information. It probably goes without saying that the internet has only made this phenomenon even more extreme.

ClickHole's approach to the category of news is basically to ask: if anything can be news, is anything news? The site distinguishes "Lifestyle" and "News" as separate categories, but to look at the content in each is to see how easily they bleed over into one another. Even as we searched for specific examples for both of these categories, we were often surprised to find that what we might consider to be News was categorized as Lifestyle and vice versa. One distinction is that while the Lifestyle category flirts with culture's fascination with celebrity, the News category, by virtue of reporting on the important people and events of the day, is able to wholly lean into this subject matter to highlight the absurdity of what we consider to be newsworthy as a society. For instance: "At It Again: Pope Francis Just Tweeted A Series Of Ambiguous Quotes About Not Being Able To Trust Your Friends," and "Heartbreaking: All The Celebrities Think That Jason Bateman Being The Emmys Valet Is Part Of A Bit That Will Pay Off In Colbert's Opening Monologue" (ClickHole, "At It Again” 2017; ClickHole, "Heartbreaking” 2017).

Other times, however, the News category pushes into more explicitly satirical territory, directly commenting on major current events. While not exactly rare, these instances are certainly less frequent than the simply absurdist material the site otherwise puts out. Stories like "Taking A Stand: Congress Has Passed A Bill Making It Illegal To Sell

${ }^{6}$ Actually two people - see (Harris \& Abidor, 2018) 
Firearms To The Las Vegas Shooter" are rare instances where the site seems to take a step back from its internet-based absurdity to connect in some way to the real world (ClickHole, "Taking A Stand" 2017). But even commentaries on real events and issues takes form in a way that is innate to the internet itself, blurring the lines of the content and its commentary in similar fashion to how the News and Lifestyle categories seem to implicitly overlap. For instance, the story " 5 Ways To Honor The Victims Of The Las Vegas Shooting Without Being Disrespectful To The NRA" (ClickHole, "5 Ways To Honor The Victims Of The Las Vegas Shooting Without Being Disrespectful To The NRA” 2017) is found in the Lifestyle category, yet is directly commenting on the internet's tendency to appropriate news and current events as a brand of lifestyle as opposed to seeing news as information that informs us about the world. In this way the site critiques a contemporary youth culture where causes and activism become conflated with identity. That this particular article takes the form of a listicle further emphasizes that this treatment of news is a phenomenon born and bred on the internet.

Voices

It is important to note that while ClickHole's headlines are the most obvious starting point for analysis, and while the site's name emerges from the concept of clickbait, the site is not merely a collection of headlines--each article on the site has actual story content associated with it. Most of this content is relatively inconsequential or even beside the point; in most instances the headline is intended to be the content. However, one area of the site that deviates from this prescription is the Voices section, which functions as a diverse collection of blog posts from numerous fictional contributors.

What distinguishes this section of the site is that the content is as much a part of the satire as the headline itself. In the Lifestyle and News sections of the site, the stories are straightforward in their presentation, in some ways not differing all that much from the similar brand of satire produced by ClickHole's parent site, The Onion. In this way, the Lifestyle and News segments may be seen as parodying and satirizing the internet's obsession with both the sensational and inconsequential. Voices, however, while sometimes putting out content that is just as seemingly random and absurd as other stories on the site (Clarke, 2017), generally functions to address trends on the internet. As a result, the content of these blog posts is implicated in the satire that this section of the site is promoting.

One example is a blog post, which mocks the culture of blogs as auto-ethnography, wherein individuals stake their claims to authenticity and individuality on aspects of themselves they see as idiosyncratic, even though such behaviors are actually rather commonplace. The epitome of this article's tone is encapsulated in one two-sentence paragraph: "In short, I can be kind of weird and dorky. And if admitting that happens to showcase what a quirky, offbeat person I am, then so be it" (Perkins, 2014). Beyond the tone of the article, its specific examples are incredibly of the cultural moment, such that the post is really aimed at calling out the artifice of the quirky, off-beat persona that many Millennials are clamoring to be seen as embodying. ${ }^{7}$

Many other articles in this section of the site function in a similar way, taking current events or even sayings and attitudes that are in vogue and highlighting their artificiality. This is done through pointing to some of the dangers implicit in a culture that tends to jump on the bandwagon for any new cause and obsesses over trends that disappear as

\footnotetext{
7 This phenomenon has been termed the "Manic Pixie Dream Girl" by some. For the origination of the term see (Rabin, 2007); for an explication of the term and its consequences for culture see (Schwyzer, 2013).
} 
quickly as they arrive. In terms of attitudes, the Voices section includes headlines like "I Just Turned 27, So I Guess It's Time to Start Describing Life as a Journey," that highlights the idea of romanticizing young adulthood (Ford, 2017). Others take on major cultural issues in ways that make fun of how everyone seems to think they have the answers to current controversies like sexual assault in Hollywood ("As A Male Feminist, I Really Think I'd Absolutely Crush It If I Ever Had To Publicly Apologize For Sexual Misconduct"), mansplaining ("Men, Stop Telling Women To Calm Down And Swim Perpendicular To The Riptide"), and police violence ("If You're Not A Police Officer, You Can't Understand The Pressure You Feel In The Split Second When You Have To Decide Whether Or Not To Shoot An Unarmed Civilian 8 Times") (Hartford, 2017; Manford, 2018; Matthews, 2018).

Quizzes

One of the most popular categories on BuzzFeed's site is Quizzes, where readers can go to ostensibly learn more about their interests, personality traits, and anything else about themselves that they might not already know. These quizzes set up a seemingly arbitrary set of conditions that the quiz's makers then use to extrapolate something supposedly significant about the quiz-taker. These quizzes problematically imply that their audience may be known entirely by the choices they make to a constrained set of options. The culture surrounding BuzzFeed's quizzes is one where people take the quizzes seriously even as they mock them. No one takes a quiz like "Buy Six School Items and We'll Give You Advice From 'Queer Eye' To Live By” (Perlman, 2018) as an actual source of life advice; these quizzes are implicitly designed to provoke conversation surrounding how much the taker of the quiz finds the results relatable.

ClickHole's quizzes do not seek to prescribe anything directly upon the taker, per se, but instead are maybe the clearest examples of how the site deconstructs the popular form they are satirizing. For instance, the quiz "How Many of These Ways Have You Padded out the Running Time of Your Brother's Funeral?" (ClickHole, "How Many of These Ways Have You Padded out the Running Time of Your Brother's Funeral?" 2018) succeeds in its absurdity and critique by implying that not only is padding the runtime of your brother's funeral something that everybody has done, but that that act in itself is something that happens so frequently that there can be multiple common ways to have done so. Even the ideas that the quiz offers--"Ask everyone in attendance to chant your brother's SAT score for 30 minutes," or "Had everyone form a line and sign their name on your brother's body."-are certainly not things that anyone has ever plausibly done at any funeral ever. This trifecta of distancing from reality is one way in which ClickHole's quizzes emphasize the arbitrary nature of the internet quiz phenomenon.

Another example may be found in the straightforwardly-named quiz, "Are You Addicted to Coffee" (ClickHole, "Are You Addicted To Coffee?" 2015)? Even when ClickHole is addressing a topic that seems rather simple, their approach is to highlight the inanity of the arbitrary criteria common in quizzes like these. Options in this quiz include: "My mother no longer speaks to me because I gave my father's eulogy while wearing a T-shirt that said 'I'm Just An Old Curmudge-on Who Loves To Get His Sludge On," and "There is a movie about my life called Often: The Frequency of Coffee." These are, to be fair, coffee related, yet so distorted from anyone's actual experience that they effectively tell you nothing about your interest in coffee. Additionally, the end result that the quiz gives implies that there are correct answers and that the quiz has an agenda by chiding those who the results say are not addicted or only a little bit addicted to "the hot black bean." The implication is that the end results of these quizzes have greater 
significance beyond the confines of the quiz. This goes back to the notion that these quizzes imply that their takers may be actively defined through the answers they give. ClickHole's quizzes actively usurp the ostensible authority of the quiz by telling the taker the actual right answers to what appear to be subjective polls.

\section{Videos}

As has already been mentioned, the content strategy that BuzzFeed pursues with video is that narrative is unnecessary (Allocca, 2018, p. 43). Instead, videos are broken up into multiple segments with the idea that viewers will relate to at least one of the segments and potentially share the content with others. The topics approached by BuzzFeed's video creators have to hit a sweet spot with the topics they address in order to be at least somewhat relatable to the largest possible audience, which is the only way that they will garner views and shares, which we have already noted is the only metric BuzzFeed puts any weight on (Allocca, 2018, p. 42).

ClickHole's response to this trend is to take their videos in the complete opposite direction. For one thing, their videos, while still typically rather short, are usually focused on one single topic or concept, and even when their videos aim for some semblance of relatability they still work to deviate from audience expectations. The video "Americans and Guzmer Try British Candy For The First Time" features a very standard BuzzFeed setup of normal American people trying foreign candy, but changes the formula by also featuring a one-eyed Jabba the Hutt-like alien who is also trying the candy, and is prominently featured sucking the candy bars down his throat (ClickHole, "Americans And Guzmer Try British Candy For The First Time" 2016).

Additionally, many of ClickHole's video topics actively pursue a type of unrelatability to viewers through disregarding popular trends or pushing away from normality. Their tendency to subvert trends seen in other videos may be glimpsed in videos like "We Couldn't Reunite The Cast Of 'The Office,' So We Just Had All These People Walk Into A Room One At A Time While The Theme Song Played" (ClickHole, "We Couldn't Reunite The Cast Of 'The Office,' So We Just Had All These People Walk Into A Room One At A Time While The Theme Song Played" 2015). Other times, ClickHole's videos stand entirely alone and are not tethered to anything audiences may be familiar with; examples here include "Heartbreaking: Unmarried Woman Brings A Tire To Take Your Daughter To Work Day," and "Not A Good Sign: This Zookeeper is Hauling Ass" (ClickHole, "Heartbreaking" 2017; ClickHole, "Not A Good Sign" 2018). Such videos take a singular concept and run with it, taking such absurdity seriously and playing it straight to effectively create an anti-joke that simultaneously subverts and emphasizes the humor in the ridiculous situations the concepts set up.

\section{ClickVentures}

ClickVentures are the most unique aspect of ClickHole's canon in that they do not directly parody anything that BuzzFeed or any similar sites are doing. The closest analog would be the "choose-your-own-adventure" book format, interestingly tying the section of the site to something outside of the digital realm. A typical ClickVenture places you, the web surfer, into a particular role in a scenario with a challenge implied in the ClickVenture's title. Each section of the ClickVenture offers you a description of the situation you are in, as well as options for action or dialog that then opens up further possibilities by moving you through the narrative. However, as may be expected given the site's predilections, even ostensibly normal-sounding ClickVentures quickly subvert expectations and go entirely off the rails. 


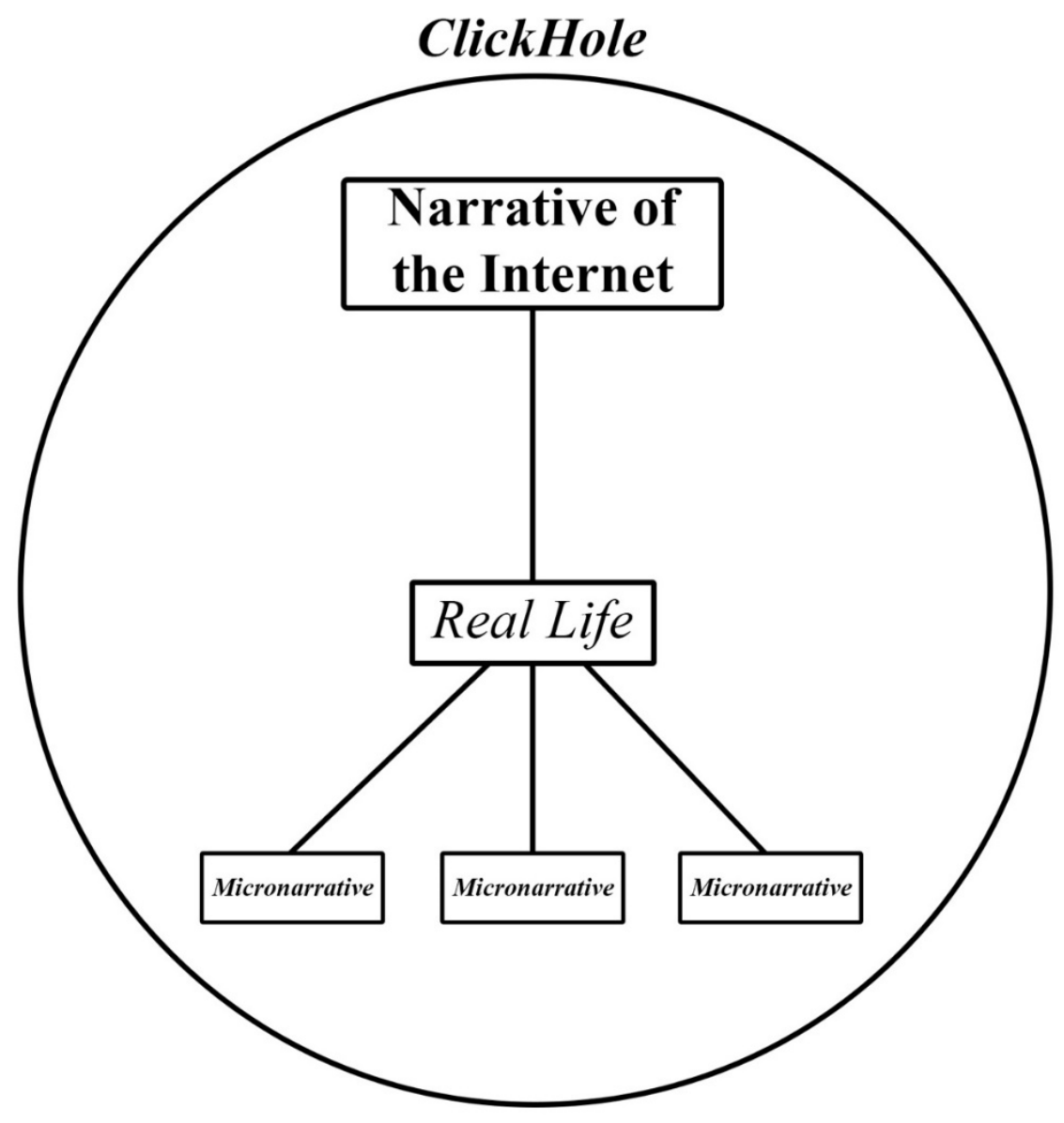

Figure 2. ClickHole and the narrative structure of the internet

One example here may be found in the mundanely titled "It's 2:51 A.M. Can You Fall Asleep" (ClickHole, "It's 2" 2017)? The ClickVenture starts by giving you the single option of lying down before allowing you to either "Toss" or "Turn." The absurdity ramps up extensively from there, eventually leading you down a potential path that can end with you abandoning corporate America to move to a monastery (amongst other things). ClickVentures are both deep in execution and ridiculous in both concept and thoroughness. The type of absurdity contained in these adventures are consistent with the themes and tone of the rest of the website, but effectively stand alone as a mode of expressing that kind of humor. These ClickVentures, then, function as a kind of overflow of the style and essence of ClickHole's entire site, transforming the narrative from one of direct parody and satire to one of originality. ClickVenture writer Anthony Easton acknowledges that "ClickVenture in a way is the essence of what ClickHole is.... You have this engrossing thing that a lot of times is nonsensical and insane, but you can't stop clicking" (Muncy, 2016).

With the inclusion of this section, ClickHole is ostensibly saying that their style of humor does not entirely rely on making fun of the form or content of other sites. Instead, its absurdity and randomness champions itself as the style of humor for the internet as a whole. Even as other sites like Reddit function as aggregates of disparate types of content, including absurdist and random humor, ClickHole's in-house production of ClickVentures (and all their narrative content) furthers the idea of the site as a master narrative of the story and form of the internet, as opposed to being defined by the whims of users. 
Parody and satire have often been themselves critiqued for their inability to offer constructive criticism to the causes or ideas that they lampoon (Fife, 2016). Though it may appear on the surface as a site with the explicit intention of parodying and satirizing internet trends, our argument is that ClickHole is in fact only using elements of parody and satire in its content in order to help construct its larger narrative. The actual form of the site is not parodying or satirizing anything but is rather a natural outgrowth of the medium of the internet. Although it may seem like it is ClickHole's intention to confuse or mislead its readers, this is not actually the case. Editor-in-chief Matt Powers notes that

It's never our goal to trick people into thinking we are a real internet aggregator. We throw away tons of jokes because we're worried they are too close to something a real, non-satirical site might actually run. A better reaction is when our readers get a cathartic laugh from one of our articles that clearly hits on something frustrating or dumb that is mindlessly repeatedly a million times on the internet. There is a role for internet aggregators and there is a role for us, and I think it's best when those don't become muddied. (Colon, 2015)

What Powers' perspective indicates is that while occasionally their content may become confused with events in the real world, hopefully most of the content ClickHole produces is too extreme or absurd to be taken seriously.

This indicates that as an entity formed entirely around the culture of the internet, the purpose of ClickHole's grand narrative is to remind the reader through a type of frustrating comparison that the internet's standard for journalism turns the mundane into the incredible. What ClickHole implicitly offers by comparison is a space for the incredible and absurd to be what they are, while leaving the mundane in its place in real life. In this way the site's narrative is arguably therapeutic and built around the idea of comforting readers in the anxious age of the internet. Former editor-in-chief Jermaine Affonso identifies this as the site's defining characteristic, saying, "I think the thesis of ClickHole is roughly: The world is a scary and horrifying place, and we're all just kind of trying to make it through" (Cox, 2015). This sentiment differentiates ClickHole from your average satire and even its parent site, The Onion, and reiterates its place as a product wholly of the internet. The stories ClickHole tells not only emerge from the internet but could arguably only be told on the internet. Whatever its relation to real-life through referencing current events or popular celebrities, the site marks a departure from a reliance upon the real world for its ideas and content and is instead based entirely upon the internet's perception of the real world. As such, the structure of its narrative is entirely unique to the internet and establishes a paradigm for what defines a truly internet-based narrative.

\section{REFERENCES}

Allocca, K. (2018). Videocracy: How YouTube Is Changing the World . . . with Double Rainbows, Singing Foxes, and Other Trends We Can't Stop Watching. New York: Bloomsbury USA.

Auwal, A. M. (2016). The Complexities of New Media: Can the 'Web Media' Completely Erase Traditional Media from the Communication Industry? Online Journal of Communication and Media Technologies, 6(3), 173-195.

Boorstin, D. J. (2012). The image: a guide to pseudo-events in America. New York: Vintage Books, a division of Random House, Inc. 
Carr, N. (2011). The shallows: what the Internet is doing to our brains. New York: W.W. Norton.

Clarke, J. (2017, October 19). I'm Starting To Worry My Prince Charming Will Never Drive Up To My House In His Homemade Station Wagon With His Mom In The Backseat, Honking His 'La Cucaracha' Horn And Waving His 9-Foot-Long Arms. Retrieved on April 17, 2018 from https://voices.clickhole.com/i-m-starting-to-worry-my-princecharming-will-never-dri-1825124245

clickbait | Definition of clickbait in English by Oxford Dictionaries. (n.d.). Retrieved on April 10, 2018 from https://en.oxforddictionaries.com/definition/clickbait

ClickHole. (2015a, January 14). We Couldn't Reunite The Cast Of 'The Office,' So We Just Had All These People Walk Into A Room One At A Time While The Theme Song Played. Retrieved on April 17, 2018 from https://www.clickhole.com/we-couldn-treunite-the-cast-of-the-office-so-we-jus- 1825126265

ClickHole. (2015b, April 20). Are You Addicted To Coffee? Retrieved on April 17, 2018 from https://quizzes.clickhole.com/are-you-addicted-to-coffee-1825124659

ClickHole. (2015c, May 19). 6 Times Tobey Maguire Had A Public Meltdown Because Nobody Would Call Him 'Tugboat Maguire.' Retrieved on April 17, 2018 from https://news.clickhole.com/6-times-tobey-maguire-had-a-public-meltdown-becausenob-1825121319

ClickHole. (2016, September 28). Americans And Guzmer Try British Candy For The First Time. Retrieved on April 17, 2018 from https://www.clickhole.com/americans-andguzmer-try-british-candy-for-the-first-ti-1825126518

ClickHole. (2017a, January 5). It's 2:51 A.M. Can You Fall Asleep? Retrieved on April 19, 2018 from https://clickventures.clickhole.com/it-s-2-51-a-m-can-you-fall-asleep1825124399

ClickHole. (2017b, June 13). Hard To Watch: Chris Pine Accompanied This Terminally Ill Girl To Prom But Was Upstaged When Chris Pratt Accompanied An Even More Terminally Ill Girl To The Same Prom. Retrieved on April 17, 2018 from https://lifestyle.clickhole.com/hard-to-watch-chris-pine-accompanied-this-terminally -i-1825120809

ClickHole. (2017c, June 27). At It Again: Pope Francis Just Tweeted A Series Of Ambiguous Quotes About Not Being Able To Trust Your Friends. Retrieved on April 17, 2018 from https://www.clickhole.com/at-it-again-pope-francis-just-tweeted-aseries-of-ambi-1825122770

ClickHole. (2017d, July 13). Heartbreaking: Unmarried Woman Brings A Tire To Take Your Daughter To Work Day. Retrieved on April 17, 2018 from https://www.clickhole.com/heartbreaking-unmarried-woman-brings-a-tire-to-take-yo $-1825126623$

ClickHole. (2017e, September 17). Heartbreaking: All The Celebrities Think That Jason Bateman Being The Emmys Valet Is Part Of A Bit That Will Pay Off In Colbert's Opening Monologue. Retrieved on April 17, 2018 from https://news.clickhole.com/heartbreaking-all-the-celebrities-think-that-jason-bat1825123744

ClickHole. (2017f, October 2). 5 Ways To Honor The Victims Of The Las Vegas Shooting Without Being Disrespectful To The NRA. Retrieved on April 17, 2018 from https://lifestyle.clickhole.com/5-ways-to-honor-the-victims-of-the-las-vegas-shootingw-1825121152 
Online Journal of Communication and Media Technologies, 2018

ClickHole. (2017g, October 3). Taking A Stand: Congress Has Passed A Bill Making It Illegal To Sell Firearms To The Las Vegas Shooter. Retrieved on April 17, 2018 from https://news.clickhole.com/taking-a-stand-congress-has-passed-a-bill-making-it-il1825122452

ClickHole. (2018a, February 7). Not A Good Sign: This Zookeeper Is Hauling Ass. Retrieved on April 22, 2018 from https://www.clickhole.com/not-a-good-sign-thiszookeeper-is-hauling-ass-1825126706

ClickHole. (2018b, February 22). Whoa: Morgan Brought Coffee to First Period. Retrieved on April 17, 2018 from https://lifestyle.clickhole.com/whoa-morgan-brought-coffee-tofirst-period-1825121677

ClickHole. (2018c, April 4). How Many Of These Ways Have You Padded out The Running Time Of Your Brother's Funeral? Retrieved on April 17, 2018 from https://quizzes.clickhole.com/how-many-of-these-ways-have-you-padded-out-therunning-1825124993

ClickHole. (2018d, April 16). This Bitch: Of Course Margot's Only Skin Routine Is Splashing Her Face with Water at Night. Retrieved on April 17, 2018 from https://www.clickhole.com/this-bitch-of-course-margot-s-only-skin-routine-is-spl1825290510

Colon, D. (2015, February 16). How'd you get that cool job at ClickHole? Retrieved on April 19, 2018 from https://brokelyn.com/howd-get-cool-job-matt-powers-getting-jobwriting-nonsense-clickhole/

Cox, A. M. (2015, July 30). Jermaine Affonso Can't Escape the Garbage, Either. The New York Times. Retrieved from https://www.nytimes.com/2015/08/02/magazine/jermaine -affonso-cant-escape-the-garbage-either.html

Edidin, R. (2014, January 8). 5 Reasons Listicles Are Here to Stay, and Why That's OK. Retrieved on April 10, 2018 from https://www.wired.com/2014/01/defense-listicle-listarticle/

Fife, J. (2016). Peeling The Onion: Satire and the Complexity of Audience Response. Rhetoric Review, 35(4), 322-334. https://doi.org/10.1080/07350198.2016.1215000

Fisher, M. (n.d.). Who cares if it's true? Retrieved on April 10, 2018 from http://www.cjr.org/cover_story/who_cares_if_its_true.php

Ford, D. (2017, March 29). I Just Turned 27, So I Guess It's Time to Start Describing Life as a Journey. Retrieved on April 17, 2018 from https://voices.clickhole.com/i-justturned-27-so-i-guess-it-s-time-to-start-describ-1825124206

Green, M. R., \& Walters, L. M. (2013). Digital Storytelling Viewed though a Post-process Lens: Reflections from the Fulbright-Hays 2010 China Seminar. Online Journal of Communication and Media Technologies, 3(1), 75-90.

Greenberg, R. (2014, June 18). When it comes to Onion's new satirical site ClickHole, take the bait. Washington Post. Retrieved from https://www.washingtonpost.com/express/ wp/2014/06/18/when-it-comes-to-onions-new-satirical-site-clickhole-take-the-bait/

Hamblin, J. (2014, November 11). It's everywhere, the Clickbait. The Atlantic. Retrieved from https://www.theatlantic.com/entertainment/archive/2014/11/clickbait-what-is/ $382545 /$

Harris, K., \& Abidor, J. (2018, April 17). Amy Schumer Played with Puppies While Answering Fan Questions and It's Hilarious. Retrieved on April 17, 2018 from https://www.buzzfeed.com/kristinharris/amy-schumer-i-feel-pretty-puppy-interview 
Hartford, B. (2017, November 17). As A Male Feminist, I Really Think I'd Absolutely Crush It If I Ever Had to Publicly Apologize for Sexual Misconduct. Retrieved on April 17, 2018 from https://voices.clickhole.com/as-a-male-feminist-i-really-think-i-dabsolutely-crush-1825124257

Isaac, M. (2014, August 10). 50 Million New Reasons BuzzFeed Wants to Take Its Content Far Beyond Lists. The New York Times. Retrieved from https://www.nytimes.com/2014/08/11/technology/a-move-to-go-beyond-lists-forcontent-at-buzzfeed.html

Jolles, D. (2014, February 17). 5 Ways Buzzfeed Is Making You Stupid. Retrieved on April 10, 2018 from http://www.techly.com.au/2014/02/17/5-ways-buzzfeed-making-stupid/

Kois, D. (2015, June 8). Wow. ClickHole. Slate. Retrieved from http://www.slate.com/articles/arts/culturebox/2015/06/clickhole_writers_explain_the ir_craft_how_the_onion_s_internet_parody_spinoff.html

Lafrance, A. (2012, August 21). BuzzFeed with a press pass: What happens when the GIF kings try to take Washington? Retrieved on April 10, 2018 from http://www.niemanlab.org/2012/08/buzzfeed-with-a-press-pass-what-happens-whenthe-gif-kings-try-to-take-washington/

Lamar, A. (2015, March 8). What is a Lifestyle Blog? Plus Categories and POV's to Get You Started. Retrieved on April 17, 2018 from http://www.honeyandpine.co/what-is-alifestyle-blog/

Manford, L. (2018, March 21). Men, Stop Telling Women To Calm Down And Swim Perpendicular To The Riptide. Retrieved on April 17, 2018 from https://voices.clickhole.com/men-stop-telling-women-to-calm-down-and-swimperpendic-1825124281

Matthews, J. (2018, April 6). If You're Not A Police Officer, You Can't Understand The Pressure You Feel In The Split Second When You Have To Decide Whether Or Not To Shoot An Unarmed Civilian 8 Times. Retrieved on April 17, 2018 from https://voices.clickhole.com/if-you-re-not-a-police-officer-you-can-t-understand-th1825124280

Muncy, J. (2016, March 26). How ClickHole Crafts the Web's Most Hilarious Adventure Games. Retrieved on April 19, 2018 from https://www.wired.com/2016/03/clickholeadventure-games/

Okrent, A. (2014, February). The listicle as literary form. Retrieved on April 10, 2018 from https://mag.uchicago.edu/arts-humanities/listicle-literary-form

Perkins, A. (2014, November 24). And If This Self-Effacing Blog Post Highlights My Appealing Quirks, So Be It. Retrieved on April 10, 2018 from http://www.clickhole.com/blogpost/and-if-self-effacing-blog-post-highlights-my-appea $-1470$

Perlman, R. (2018, April 14). Buy Six School Items and We'll Give You Advice from "Queer Eye" To Live By. Retrieved on April 17, 2018 from https://www.buzzfeed.com/rileyp411d4e452/shop-for-school-supplies-and-we-willgive-you-advi-2h5gx

Postman, N. (1993). Technopoly: the surrender of culture to technology. New York: Vintage Books.

Rabin, N. (2007, January 25). The Bataan Death March of Whimsy Case File \#1: Elizabethtown. Retrieved on April 17, 2018 from https://film.avclub.com/the-bataandeath-march-of-whimsy-case-file-1-elizabet-1798210595 
Online Journal of Communication and Media Technologies, 2018

Schwyzer, H. (2013, July 9). The Real-World Consequences of the Manic Pixie Dream Girl Cliché. The Atlantic. Retrieved from https:/www.theatlantic.com/sexes/archive/2013/ 07/the-real-world-consequences-of-the-manic-pixie-dream-girl-clich-233/277645/

Shepherd, J. (2012, May 30). 40 of the Most Powerful Photographs Ever Taken. Retrieved on April 10, 2018 from https://www.buzzfeed.com/expresident/most-powerfulphotographs-ever-taken

Spangler, T. (2017, April 26). BuzzFeed's Latest Viral Craze: Ex-Staffers Bashing the Company on YouTube. Retrieved on April 12, 2018 from http://variety.com/2017/digital/news/why-i-left-buzzfeed-youtube-1202399091/

Tandoc, E. C., \& Jenkins, J. (2017). The Buzzfeedication of journalism? How traditional news organizations are talking about a new entrant to the journalistic field will surprise you! Journalism, 18(4), 482-500. https://doi.org/10.1177/1464884915620269

The Inverted Pyramid Structure. (n.d.). Retrieved on April 12, 2018 from https://owl.english.purdue.edu/owl/resource/735/04/

Torres, K. (2018, April 15). This Is What People Are Referring To Their Periods As These Days. Retrieved on April 17, 2018 from https://www.buzzfeed.com/kristatorres/101weirdly-funny-nicknames-people-use-when-its-that-time

What Is ClickHole? (2014, June 12). Retrieved on April 10, 2018 from http://www.clickhole.com/article/what-clickhole-230

Yandoli, K. (2014, June 20). 15 Poop Horror Stories That Will Make You Feel Better About Yourself. Retrieved on April 10, 2018 from https://www.buzzfeed.com/kaylayandoli/ hurbly-grblies

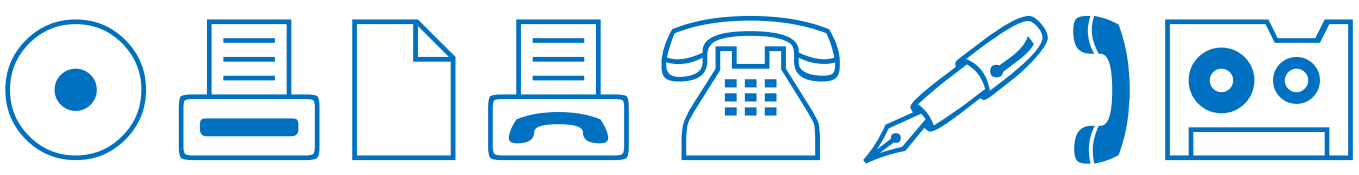

